\title{
Unexpected dielectric response in lead zirconate titanate ceramics: The role of ferroelectric domain wall pinning effects
}

\author{
J. E. Garcia, V. Gomis, R. Perez, A. Albareda, and J. A. Eiras
}

Citation: Appl. Phys. Lett. 91, 042902 (2007); doi: 10.1063/1.2759983

View online: https://doi.org/10.1063/1.2759983

View Table of Contents: http://aip.scitation.org/toc/apl/91/4

Published by the American Institute of Physics

\section{Articles you may be interested in}

Strong ferroelectric domain-wall pinning in $\mathrm{BiFeO}_{3}$ ceramics

Journal of Applied Physics 108, 074107 (2010); 10.1063/1.3490249

Oxygen-vacancy ordering as a fatigue mechanism in perovskite ferroelectrics

Applied Physics Letters 76, 3801 (2000); 10.1063/1.126786

Effect of Mn-acceptor dopant on dielectric and piezoelectric responses of lead lanthanum zirconate titanate piezoceramics

Journal of Applied Physics 110, 034106 (2011); 10.1063/1.3622338

Ferroelectric thin films: Review of materials, properties, and applications

Journal of Applied Physics 100, 051606 (2006); 10.1063/1.2336999

Ultrahigh strain and piezoelectric behavior in relaxor based ferroelectric single crystals Journal of Applied Physics 82, 1804 (1997); 10.1063/1.365983

Evidence of temperature dependent domain wall dynamics in hard lead zirconate titanate piezoceramics Journal of Applied Physics 112, 014113 (2012); 10.1063/1.4736582

\section{AIP Conference Proceedings}

\section{Get $30 \%$ off all}

Enter Promotion Code PD/F-30 at checkout print proceedingsl 


\title{
Unexpected dielectric response in lead zirconate titanate ceramics: The role of ferroelectric domain wall pinning effects
}

\author{
J. E. Garcia, ${ }^{\text {a) }}$ V. Gomis, R. Perez, and A. Albareda \\ Department of Applied Physics, Universitat Politècnica de Catalunya, 08034 Barcelona, Spain \\ J. A. Eiras \\ Physics Department, Universidade Federal de São Carlos, CEP 13565-905 Sao Carlos-SP, Brazil
}

(Received 16 March 2007; accepted 24 June 2007; published online 24 July 2007)

\begin{abstract}
Temperature dependent dielectric response has been measured in $\mathrm{Pb}\left(\mathrm{Zr}_{1-x} \mathrm{Ti}_{x}\right) \mathrm{O}_{3}$ ceramics. Samples of different compositions $(x=0.40,0.47$, and 0.60$)$, pure and doped with $\mathrm{Nb}$-or Fe, were studied at temperatures between 15 and $700 \mathrm{~K}$ and in the frequency range from $100 \mathrm{~Hz}$ to $1 \mathrm{MHz}$. Unexpected dielectric behavior has been found around room temperature. Anomalous temperature dependent permittivity is observed in pure and Fe-doped samples but not in Nb-doped samples. The anomaly appears related to the presence of oxygen vacancies but not on the sample crystallographic phase. The authors suggest that the anomaly may be a manifestation of the domain wall pinning effect. (C) 2007 American Institute of Physics. [DOI: 10.1063/1.2759983]
\end{abstract}

$\mathrm{Pb}\left(\mathrm{Zr}_{1-x} \mathrm{Ti}_{x}\right) \mathrm{O}_{3}(\mathrm{PZT})$ based ferroelectric materials have been widely studied due to their large number of applications, especially those at morphotropic phase boundary (MPB). ${ }^{1-3}$ Although their phase diagram and general properties are well established, a complete modeling for understanding their physical properties is still lacking. It is well known that the substitution of $\mathrm{Zr}^{4+}$ or $\mathrm{Ti}^{4+}$ with pentavalent or trivalent cations largely modifies their physical properties. ${ }^{3}$ The dielectric response at room temperature is mainly due to domain wall motion (extrinsic contribution) in these materials. ${ }^{4}$ Defects as the complex defects generated by oxygen vacancies can act as effective pinning centers to the domain wall motion. ${ }^{5,6}$ As a consequence, different permittivity values for the same $\mathrm{Zr} / \mathrm{Ti}$ ratio are found in pure, $\mathrm{Nb}$-doped, and Fe-doped materials. Monotonous decreasing $\varepsilon^{\prime}(T)$ would be produced in any case, due to the domain wall freezing when samples are cooled down, reaching intrinsic dielectric constant at very low temperatures. Nevertheless, the experimental results reveal more complex behavior. We present a study of the temperature dependent dielectric permittivity which shows a plateaulike dependence around room temperature in pure and Fe-doped samples. The influence of the composition and crystallographic structure is analyzed. The results are discussed within the framework of the ferroelectric domain wall dynamics.

$\mathrm{Pb}\left(\mathrm{Zr}_{1-x} \mathrm{Ti}_{x}\right) \mathrm{O}_{3}$ samples were prepared by solid state reaction of different stoichiometric mixtures of $\mathrm{PbO}, \mathrm{Zr}_{2} \mathrm{O}_{3}$, and $\mathrm{TiO}_{2}$. Precursors were mixed in a ball mill during $2 \mathrm{~h}$, dried at $80{ }^{\circ} \mathrm{C}$, and calcined at $850{ }^{\circ} \mathrm{C}$ for $3.5 \mathrm{~h}$. Different compositions of $\mathrm{Pb}\left(\mathrm{Zr}_{1-x} \mathrm{Ti}_{x}\right) \mathrm{O}_{3}$ with $x=0.40$ (PZT40), 0.47 (PZT47), and 0.60 (PZT60) were prepared. Nb- (PNZT40, PNZT47, and PNZT60) and Fe-doped (PFZT40, PFZT47, and PFZT60) powders were prepared by additing $1 \mathrm{wt} \%$ of $\mathrm{Nb}_{2} \mathrm{O}_{5}$ or $\mathrm{Fe}_{2} \mathrm{O}_{3}$ to the green moisture before homogenization by a second ball milling. Powders were uniaxially pressed at $100 \mathrm{MPa}$ into pellets of $20 \mathrm{~mm}$ in diameter and about $5 \mathrm{~mm}$ length and then isostatically pressed at $120 \mathrm{MPa}$. Sintering was carried out in a saturated $\mathrm{PbO}$ atmo-

\footnotetext{
${ }^{a)}$ Electronic mail: jose@fa.upc.edu
}

sphere. Samples were cut into disks of $15-16 \mathrm{~mm}$ in diameter and $0.8-0.9 \mathrm{~mm}$ in thickness, avoiding $\mathrm{Pb}$ inhomogeneous areas. Average grain size between 3.0 and $3.5 \mu \mathrm{m}$ was found by scanning electron microscopy. X-ray diffraction analysis revealed the expected crystallographic phase: rhombohedral for $x=0.40$, tetragonal for $x=0.60$, and MPB for $x$ $=0.47$. Neither spurious phases nor contaminants were detected. Silver electrodes were painted on both sides of the disks. After polishing, organic solvents were removed by heat treatment at $600{ }^{\circ} \mathrm{C}$ for $30 \mathrm{~min}$, which also release the induced mechanical stress during polishing. Dielectric measurements were carried out in a plane capacitor configuration at the Department of Applied Physics of UPC (Spain) and at the Physics Department of UFSCar (Brazil). Experimental setup was placed in a closed loop cold finger cryogenic systems for a measurements from 15 up to $700 \mathrm{~K}$. Dielectric response was measured using Agilent 4294A and HP-4194A impedance analyzers at frequencies from $100 \mathrm{~Hz}$ to $1 \mathrm{MHz}$.

Dielectric constant $\varepsilon^{\prime}(T)$ is displayed in Figs. 1(a) and 1(b) for PFZT47 and PNZT47 (Fe- and Nb-doped samples at MPB). In both cases, $\varepsilon^{\prime}(T)$ increases from an intrinsic low temperature dielectric constant close to 350 reaching the ferro-paraelectric transition at around $600 \mathrm{~K}$ (see insets of Fig. 1). Interesting features can be observed if we focus our attention at temperatures just below room temperatures (Fig. 1). For PFZT47, $\varepsilon^{\prime}(T)$ displays a plateaulike between 150 and $300 \mathrm{~K}$ and a kink at $300 \mathrm{~K}$. The temperature dependence of $\varepsilon^{\prime}(T)$ smoothes out above $150 \mathrm{~K}$ and becomes near temperature independent close to $300 \mathrm{~K}$ above which $\varepsilon^{\prime}(T)$ increases his dependence. This result contrasts with the response of PNZT47, which shows a "normal" monotonous increasing $\varepsilon^{\prime}(T)$ dependence at the same temperatures. We found qualitatively analogous results in experiments carried out in hard and soft commercial PZT samples (Pz26 and Pz27, respectively, from Ferroperm S/A).

Similar behavior was reported by Zhang et al. ${ }^{4}$ in 1994. The effect, named "broad anomaly," was detected for temperatures above $260 \mathrm{~K}$ in pure bulk PZT at MPB. In contrast, no anomalous response was observed in commercial soft PZT. Anomalous behavior was also reported by Sheen and $\mathrm{Kim}^{7}$ in PZT thin films for compositions also in the MPB. 


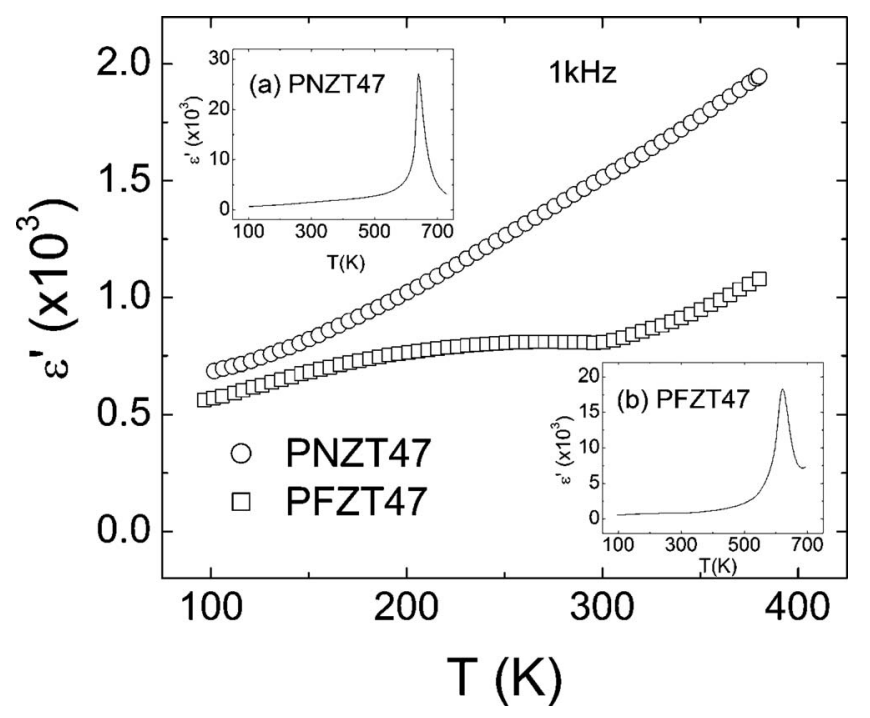

FIG. 1. Temperature dependent dielectric constant $\varepsilon^{\prime}(T)$ for samples at MPB doped with $\mathrm{Nb}^{5+}$ (PNZT47) and $\mathrm{Fe}^{3+}$ (PFZT47). Unusual behavior is found in Fe-doped sample. Nearly constant $\varepsilon^{\prime}(T)$ can be observed between 150 and $300 \mathrm{~K}$ when compared with the expected response shown by the $\mathrm{Nb}$-doped sample. Complete temperature dependence is shown at the insers.

The anomaly was detected in both $\varepsilon^{\prime}(T)$ and $\varepsilon^{\prime \prime}(T)$. These authors associate the effect to the coexistence of tetragonal and monoclinic or rhombohedral phases at MPB. A random distribution of the two types of polar clusters may produce a dipole glassylike behavior similar to the experimentally observed. Our results agree with these previously reported with the exception of the donor-doped samples, as it was also observed by Zhang et $a l^{4}$ The effect, localized at the tem- peratures between 150 and $300 \mathrm{~K}$ in $\mathrm{Fe}$-doped samples, could be associated with the coexistence of tetragonalrhombohedral phases ${ }^{8}$ or to the existence of the monoclinic phase for compositions near MPB. ${ }^{9}$ In order to clarify such a hypothesis, $\varepsilon^{\prime}(T)$ and $\varepsilon^{\prime \prime}(T)$ were further investigated in pure tetragonal and rhombohedral single phase samples [Fig. 2(a) and 2(b)]. As can be observed, the anomaly in $\varepsilon^{\prime}(T)$ was detected in both samples although their compositions are far away from MPB. Simultaneously, $\varepsilon^{\prime \prime}(T)$ displays a dissipation peak similar to those reported by Sheen and Kim. ${ }^{7}$ However, in our case, the effect cannot be directly associated with PZT crystallographic phase coexistence or ferroelectricferroelectric phase transition as was proposed for samples near MPB. ${ }^{7}$ Further measurements were carried out in Feand $\mathrm{Nb}$-doped samples in both tetragonal and rhombohedral single phases. The anomaly was also observed and even becomes more important in Fe-doped samples, independently of the particular sample crystallographic phase [Figs. 2(b) and 2(e)]. However, the anomaly was again not observed in $\mathrm{Nb}$-doped samples both in rhombohedral [Fig. 2(c)] and in tetragonal [Fig. 2(f)] single phases. We can conclude that donor doping clearly suppresses the effect. The results clearly demonstrate that doping plays a prominent role.

The anomaly appears in pure and becomes more important in acceptor-doped samples, in contrast with the behavior found in donor-doped samples. Acceptor or donor doping largely modifies the formation of oxygen vacancies already present in pure PZT. The formation of oxygen vacancy seems to be inevitable in PZT due to Pb evaporation during the processing. The formation of $\mathrm{Pb}$ vacancies, experimentally verified ${ }^{10}$ and also modelized, ${ }^{11}$ may cause the creation
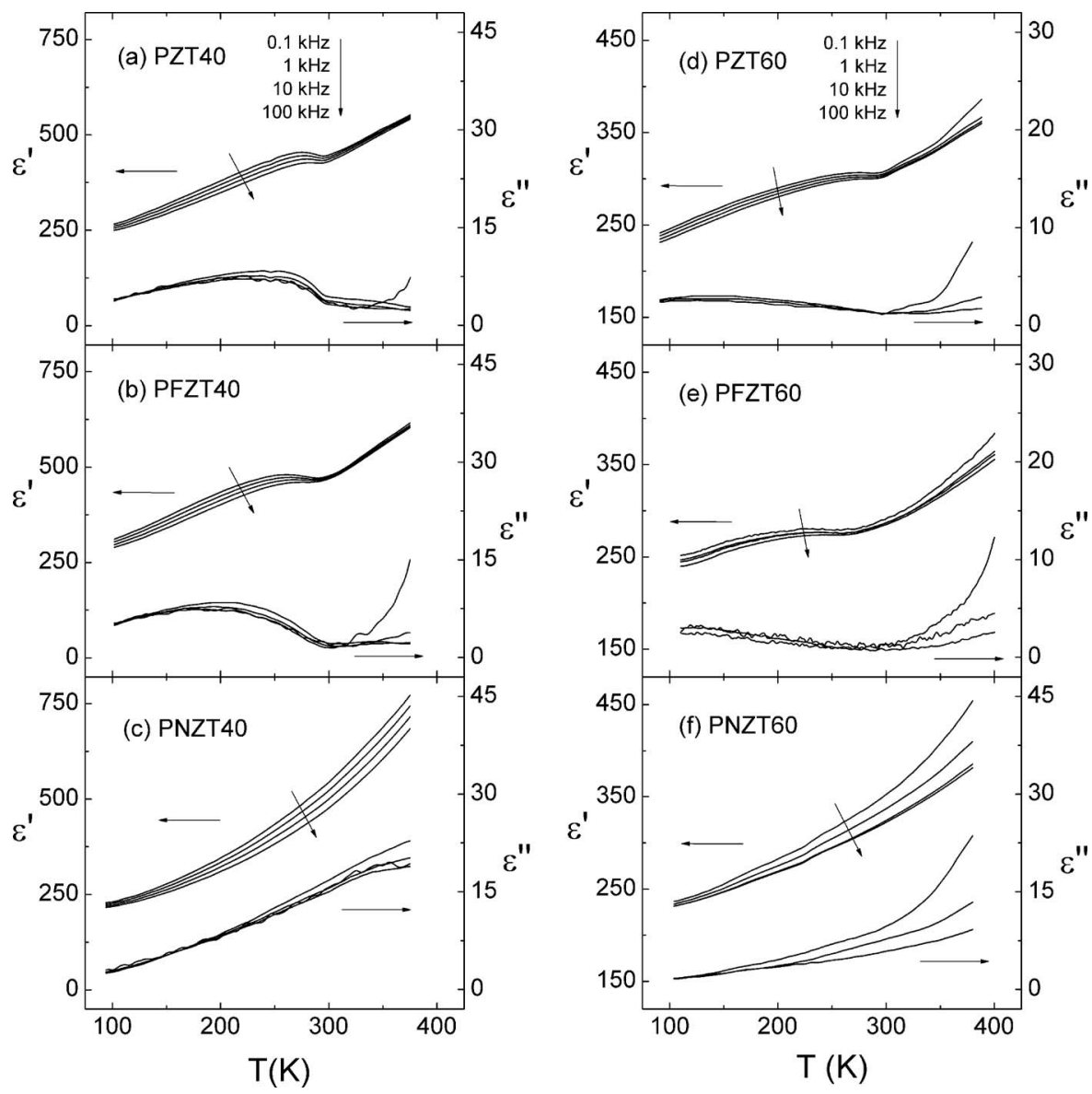

FIG. 2. Dielectric constant $\varepsilon^{\prime}(T)$ and dielectric loss $\varepsilon^{\prime \prime}(T)$ for different $\mathrm{Pb}\left(\mathrm{Zr}_{1-x} \mathrm{Ti}_{x}\right) \mathrm{O}_{3}$ samples: (a) pure rhombohedral, (b) Fe-doped rhombohedral, (c) Nb-doped rhombohedral, (d) pure tetragonal, (e) Fe-doped tetragonal, and (f) $\mathrm{Nb}$-doped tetragonal. The anomaly behavior in $\varepsilon^{\prime}(T)$, observed in pure PZT, is suppressed by donor doping. In contrast, acceptor doping seems to increase the effect. Associated with the anomaly in $\varepsilon^{\prime}(T)$, a dissipation maximum appears in $\varepsilon^{\prime \prime}(T)$. 
of oxygen vacancies in order to keep local electroneutrality. Reduction of oxygen vacancies can be achieved by using some donor dopands. ${ }^{12}$ The two holes caused by removing one $\mathrm{Pb}$ atom can be compensated by the substitution of $\mathrm{Zr}^{4+}$ or $\mathrm{Ti}^{4+}$ by $\mathrm{Nb}^{5+}$, preventing the formation of oxygen vacancies. Opposite effect is produced when the substitution is performed with $\mathrm{Fe}^{3+}$, increasing the oxygen vacancies. ${ }^{5,13}$ From our experimental results, it can be inferred that the dielectric anomaly is observed in samples where oxygen vacancies and their related effects are expected to be important, independently on the sample crystallographic phase.

As was pointed out by Poykko and Chadi, ${ }^{14}$ the oxygen vacancies are sufficiently mobile to form complex defects able to pin the domain walls, leading to the reduction in the domain wall mobility. ${ }^{15,16}$ The anomaly can then be understood not as the effect of a phase coexistence or phase transition but as a result of a domain wall pinning effect. The results suggest the presence of different pinning regimes with the temperature, where domain interactions, oxygen vacancies mobility, or collective pinning ${ }^{17}$ effects could be relevant.

In summary, different dielectric responses have been detected in PZT samples near room temperature. Anomalous $\varepsilon^{\prime}(T)$ and $\varepsilon^{\prime \prime}(T)$ were found in pure and acceptor-doped samples but not in donor-doped samples, for a measurements carried out in rhombohedral phase, tetragonal phase, or samples at MPB. The anomaly was found in samples where the formation of oxygen vacancies is expected independently of the particular crystallographic phase. The oxygen vacancies originate complex defects which work as effective pinning centers to the domain wall mobility. The anomaly, which appears to be intimately related with oxygen vacancies, may be a manifestation of the domain wall pinning effect. The existence of different pinning regimes with the temperature becomes the most suitable explanation for the observed behavior. In any case, further study of the observed dielectric behavior could shed new light on ferroelectric domain wall pinning mechanisms. The presence of the effect at room temperature makes itself interesting for application purposes.

This work was supported by project MAT2004-01341 of the Spanish MEC and the Brazilian agencies CAPES, CNPq, and FAPESP.

${ }^{1}$ Kenji Uchino, Piezoelectric Actuator and Ultrasonic Motors (Kluwer Academic, New York, 1997).

${ }^{2}$ Gene H. Haertling, J. Am. Ceram. Soc. 82, 797 (1999).

${ }^{3}$ B. Jaffe, W. R. Cook, and H. Jaffe, Piezoelectric Ceramics (Academic, London, 1971).

${ }^{4}$ Q. M. Zhang, H. Wang, N. Kim, and L. E. Cross, J. Appl. Phys. 75, 454 (1994).

${ }^{5}$ S. Takahashi, Ferroelectrics 41, 143 (1982).

${ }^{6}$ W. L. Warren, G. E. Pike, K. Vanheusden, D. Dimos, B. A. Tuttle, and J. Robertson, J. Appl. Phys. 79, 9250 (1996).

${ }^{7}$ Dongsun Sheen and Jong-Jean Kim, Phys. Rev. B 67, 144102 (2003).

${ }^{8}$ G. A. Rossetti, W. Zhang, and A. G. Khachaturyan, Appl. Phys. Lett. 88, 072912 (2006).

${ }^{9}$ B. Noheda, D. E. Cox, G. Shirane, R. Guo, B. Jones, and L. E. Cross, Phys. Rev. B 63, 014103 (2000).

${ }^{10}$ G. Burns and B. A. Scott, Phys. Rev. Lett. 25, 1191 (1970).

${ }^{11}$ Z. Zhang, P. Wu, L. Lu, and C. Shu, Appl. Phys. Lett. 88, 142902 (2006).

${ }^{12}$ Z. Zhang, L. Lu, C. Shu, and P. Wu, Appl. Phys. Lett. 89, 152909 (2006).

${ }^{13}$ Q. Tan, J. Li, and D. Viehland, Appl. Phys. Lett. 75, 418 (1999).

${ }^{14}$ S. Poykko and D. J. Chadi, Appl. Phys. Lett. 76, 499 (2000).

${ }^{15}$ C. H. Park and D. J. Chadi, Phys. Rev. B 57, R13961 (1998).

${ }^{16}$ A. Gruverman, O. Auciello, and H. Tokumoto, Appl. Phys. Lett. 69, 3191 (1996).

${ }^{17}$ Y. N. Huang, X. Li, Y. Ding, Y. N. Wang, H. M. Shen, Z. F. Zhang, C. S. Fang, S. H. Zhuo, and P. C. W. Fung, Phys. Rev. B 55, 16159 (1997). 